Pacific Journal of Mathematic 


\title{
CONTINUOUS LINEAR MAPS POSITIVE ON INCREASING CONTINUOUS FUNCTIONS
}

\author{
A. K. Katsaras
}

Let $E$ be a locally convex lattice and $X$ a completely regular space ordered by a closed order relation. We study $E$-valued (resp. $E^{\prime}$-valued) measures on an algebra or a $\sigma$-algebra of subsets of $X$ with respect to which every increasing continuous real (resp. $E$-valued) function with relatively compact range has positive integral.

Introduction. In 1951, Nachbin [11] proved the following Theorem: Let $X$ be a compact ordered space and $m$ a Radon measure on $X$ with respect to which each increasing continuous real-valued function on $X$ has a positive integral. Then, there exists a positive Radon measure $\mu$ on the locally compact space $G-\Delta$ (where $G$ is the graph of the order relation and $\Delta$ is the diagonal of $X \times X)$ of finite total mass $\|\mu\|=\|m\| / 2$ such that

$$
\int_{X} f d m=\int_{G-\Delta}\{f(y)-f(x)\} d \mu(x, y)
$$

for each continuous real-valued function $f$ on $X$.

The definition of Radon measure is as in Bourbaki [2]. Recently, Hommel [4] proved the same Theorem for locally compact spaces under an additional assumption which always holds for compact spaces.

In this paper, we examine the question of whether a Theorem of this type holds when the measures take values in a locally convex lattice $E$. We also look at $E^{\prime}$-valued measures, defined on an algebra or a $\sigma$-algebra of subsets of $X$, with respect to which the integral of each increasing continuous $E$-valued function on $X$, whose range is relatively compact, is positive.

1. Preliminaries. Throughout this paper, $X$ will denote a nonempty completely regular Hausdorff space ordered by a closed relation whose graph will be denoted by $G$. By $\Delta$ we will denote the diagonal set in $X \times X$. On all concepts related to ordered topological spaces we follow the terminology of Nachbin [13]. We will denote by $E$ a real locally convex Hausdorff lattice. If $f$ is an $E$-valued function on $X$, the function $f^{*}$ will be defined on $G$ by $f^{*}(x, y)=$ 
$f(y)-f(x)$. Clearly $f^{*} \geqq 0$ iff $f$ is increasing. Let $C(X, E)$ be the space of all continuous $E$-valued functions on $X$ and let $C_{r c}(X, E)$ denote the space of all $f$ in $C(X, E)$ whose range is relatively compact in $E$. We will denote $C(X, R)$ and $C_{r c}(X, R)$ by $C(X)$ and $C^{b}(X)$ respectively $(R$ is the space of reals). When $E$ is a normed space and $f$ a bounded $E$-valued function on $X$, we define

$$
\|f\|=\sup \{\|f(x)\|: x \in X\} .
$$

Let $B(X)$ denote the algebra, of subsets of $X$, generated by the zero sets (see Varadarajan [16]). By $B a(X)$ and $B o(X)$ we will denote, respectively, the $\sigma$-algebras of Baire and Borel subsets of $X$. The spaces of measures $M(X), M_{\tau}(B o(X)), M\left(B(X), E^{\prime}\right), M_{\sigma}\left(B a(X), E^{\prime}\right)$ and $M_{\tau}\left(B o(X), E^{\prime}\right)$ are as defined in [5] while the space $M_{t}\left(B o(X), E^{\prime}\right)$, of all tight members of $M_{\tau}\left(B o(X), E^{\prime}\right)$, is as defined in [7]. Integration of functions, with respect to members of the above spaces of measures, is also defined in [5] and [7]. The spaces $M\left(B(X), E^{\prime}\right), M_{\sigma}\left(B a(X), E^{\prime}\right)$ and $M_{\tau}\left(B o(X), E^{\prime}\right)$ become lattices under the order relation $m_{1} \geqq m_{2}$ iff $m_{1}(A) \geqq m_{2}(A)$ for every member $A$ of the algebra on which the measures are defined (see [5], [6] and [7]).

\section{Members of $M\left(B(X), E^{\prime}\right)$ positive on increasing} functions in $C_{r c}(X, E)$. Hommel [4] has studied properties of the Radon measures on an ordered locally compact space with respect to which every increasing real continuous function has positive integral. In this section we look at those members $m$ of $M\left(B(X), E^{\prime}\right)$ such that $\int_{X} f d m \geqq 0$ for every increasing $f$ in $C_{r c}(X, E)$. We begin with the following Theorem.

THEOREM 2.1. Let $X$ be a normally ordered space and $m$ a tight member of $M_{\tau}\left(B o(X), E^{\prime}\right)$ such that $\int f d m \geqq 0$ for each $f$ in $C_{r c}(X, E)$ increasing. Then, for each increasing Borel subset $F$ of $X$ we have $m(F) \geqq 0$ and for each decreasing member $A$ of $B o(X)$ we have $m(A) \leqq 0$.

Proof. Assume first that $F$ is an increasing closed subset of $X$ and let $s \geqq 0$ in $E$. Given $\epsilon>0$, there exists $K \subset X-F$ compact such that $|m s|((X-F)-K)<\epsilon$. By Nachbin ([13], Proposition 4), the decreasing hull $d(K)$ of $K$ is closed. Also $d(K) \subset X-F$ because $X-F$ is decreasing. Since $X$ is normally ordered, there exists (see Nachbin [13], Theorem 1) a real increasing continuous function $f$ on $X, 0 \leqq f \leqq 1$, with 
$f=1$ on $F$ and $f=0$ on $d(K)$. Now, the function $f s: X \rightarrow E$ is an increasing member of $C_{r c}(X, E)$ and hence $\int f s d m \geqq 0$. If $F_{1}=$ $[X-d(K)]-F$, then $|m s|\left(F_{1}\right) \leqq \epsilon$ and thus

$$
0 \leqq \int_{X} f s d m=\int_{F} f s d m+\int_{F_{1}} f s d m \leqq m(F) s+\epsilon .
$$

Thus $m(F) s \geqq-\epsilon$ for each $\epsilon>0$. From this follows that $m(F) s \geqq 0$ for each $s \geqq 0$ in $E$ and hence $m(F) \geqq 0$.

Suppose next that $A$ is an arbitrary increasing Borel subset of $X$. Let $s \geqq 0$ be in $E$. Given $\epsilon>0$, we can choose $K_{1} \subset A$ compact such that $|m s|\left(A-K_{1}\right)<\epsilon$. Let $F$ be the increasing hull of $K_{1}$. Then $F$ is closed and $K_{1} \subset F \subset A$. Thus $|m s|(A-F)<\epsilon$ and therefore $m(A) s>m(F) s-\epsilon \geqq-\epsilon$. It follows that $m(A) \geqq 0$.

To prove the last assertion, we first observe that, for each $s \in E$, we have

$$
m(X) s=\int_{X} s d m \geqq 0 \quad \text { and } \quad-m(X) s=\int_{X}(-s) d m \geqq 0
$$

and thus $m(X)=0$. Now the result follows from the fact that if $V$ is a decreasing subset of $X$, then $X-V$ is increasing.

THEOREM 2.2. Let $m \in M(X)$ be such that $m(X)=0$ and $m(A) \geqq$ 0 for each increasing member $A$ of $B(X)$. Then $\int f d m \geqq 0$ for every increasing member $f$ of $C^{b}(X)$.

Proof. Let $f \in C^{b}(X)$ be increasing. Since $m(X)=0$ we may assume, without loss of generality, that $0<f<1$. Let $n$ be a positive integer. For each $k$, let

$$
V_{k}=\{x \in X: f(x)>k / n\}, \quad A_{k}=V_{k}-V_{k+1} .
$$

Each $V_{k}$ is increasing and the sets $A_{0}, A_{1}, \cdots, A_{n-1}$ form a partition of $X$ into members of $B(X)$. Since $|f-k / n| \leqq 1 / n$ on $A_{k}$, we have

$$
\begin{aligned}
\left|\int_{X} f d m-\frac{1}{n} \sum_{k=0}^{n-1} k m\left(A_{k}\right)\right| & =\left|\sum_{k=0}^{n-1} \int_{A_{k}}(f-k / n) d m\right| \\
& \leqq \frac{1}{n} \sum_{k=0}^{n-1}|m|\left(A_{k}\right)=\|m\| / n
\end{aligned}
$$

Since

$$
\sum_{k=0}^{n-1} k m\left(A_{k}\right)=\sum_{k=1}^{n} m\left(V_{k}\right) \geqq 0,
$$


we have $\int f d m \geqq-\|m\| / n$. This proves that $\int f d m \geqq 0$ and the proof is complete.

THEOREM 2.3. Assume that $E$ is finite dimensional and let $m \in$ $M\left(B(X), E^{\prime}\right)$ be such that $m(X)=0$ and $m(A) \geqq 0$ for each increasing set $A \in B(X)$. Then, $\int f d m \geqq 0$ for each increasing $f$ in $C_{r c}(X, E)$.

Proof. There exists a base $e_{1}, \cdots, e_{n}$ for $E$ such that an element $x=\sum_{j=1}^{n} c_{j} e_{j}$ is in the positive cone of $E$ iff each $c_{i} \geqq 0$. Let $T_{i}: E \rightarrow R$, $x=\sum_{j=1}^{n} c_{j} e_{j} \mapsto c_{i}, i=1, \cdots, n$. Each $T_{i}$ is positive. Let now $f: X \rightarrow E$ be an increasing bounded continuous function. Each $f_{i}=T_{i} \circ f$ is an increasing member of $C^{b}(X)$ and $f=\sum_{j=1}^{n} f_{j} e_{j}$. Let $m_{j}=m e_{j}$. Then $m_{j}(X)=0$ and $m_{j}(A) \geqq 0$ for each increasing $A \in B(X)$. By Theorem 2.2, we have

$$
\int f d m=\sum_{j=1}^{n} \int f_{j} d m_{j} \geqq 0
$$

which was to be proved.

THEOREM 2.4. Let E be a Banach lattice and assume that there exists a sequence $\left\{e_{n}\right\}$ in the positive cone of $E$ and a sequence $\left\{\varphi_{n}\right\}$ in the positive cone of $E^{\prime}$ such that $x=\sum_{n=1}^{\infty} \varphi_{n}(x) e_{n}$ for every $x$ of $E$. Let $m \in M_{\sigma}\left(B(X), E^{\prime}\right)$ be such that $m(X)=0$ and $m(A) \geqq 0$ for each increasing $A \in B(X)$. Then $\int f d m \geqq 0$ for each increasing $f \in C_{r c}(X, E)$.

Proof. For each positive integer $n$, define $T_{n}: E \rightarrow E, T_{n}(x)=$ $\sum_{k=1}^{n} \varphi_{k}(x) e_{k}$. By the principle of uniform boundedness, we have that $\sup \left\{\left\|T_{n}\right\|: n=1,2, \cdots\right\}=M<\infty$. Let now $f$ be an increasing member of $C_{r c}(X, E)$. Each $f_{n}=T_{n} \circ f$ is an increasing member of $C_{r c}(X, E)$. Moreover, for each $x \in X$ we have $\left\|f_{n}(x)\right\| \leqq M\|f\|$ and $f_{n}(x) \rightarrow f(x)$. Since $m$ is $\sigma$-additive, we have

$$
\int f d m=\lim _{n \rightarrow \infty} \int f_{n} d m=\lim _{n \rightarrow \infty} \sum_{k=1}^{n} \int f_{k} e_{k} d m=\lim _{n \rightarrow \infty} \sum_{k=1}^{n} \int f_{k} d\left(m e_{k}\right) \geqq 0
$$

by Theorem 2.2. Hence the result follows.

COROLlary 2.5. Let $E=l^{p}, 1 \leqq p<\infty$, be the space of all real sequences $x=\left\{x_{n}\right\}$ for which $\|x\|_{p}=\left(\Sigma\left|x_{n}\right|^{p}\right)^{1 / p}<\infty$, ordered by the cone $P=\left\{x=\left(x_{n}\right): x_{n} \geqq 0\right.$ for each $\left.n\right\}$. If $m \in M_{\sigma}\left(B(X), E^{\prime}\right)$ is such that 
$m(X)=0$ and $m(A) \geqq 0$ for each $A \in B(X)$ increasing, then $\int f d m \geqq 0$ for each increasing $f \in C_{r c}(X, E)$.

Proof. Take $e_{n}=(0,0, \cdots, 1,0, \cdots)$ with the 1 in the $n$th position. For each $n$, let $\varphi_{n}: E \rightarrow R,\left(x_{k}\right) \mapsto x_{n}$. Now apply the preceding Theorem.

THEOREM 2.6. Let $X$ be a compact ordered space and $E=l^{p}$ (space of real sequences), $1 \leqq p<\infty$, with the usual norm and ordered by the cone $\left\{x=\left(x_{n}\right) \in l^{p}: x_{n} \geqq 0\right.$ for each $\left.n\right\}$. Let $m \in M_{\tau}\left(B o(X), E^{\prime}\right)$ be such that $\int f d m \geqq 0$ for each continuous increasing $E$-valued function $f$ on $X$. For each positive integer $n$, let $m_{n}=m e_{n}$ where $e_{n}=(0,0, \cdots, 1,0, \cdots)$ with the 1 in the nth position. If the sequence $\left\{\left\|m_{n}\right\|\right\}$ is in $l^{1}$, then there exists $0 \leqq \mu \in M_{t}\left(B o(G-\Delta), E^{\prime}\right)$ such that

$$
\int_{X} f d m=\int_{G-\Delta} f d \mu
$$

for each $f$ in $C(X, E)$.

Proof. Suppose that $\left\{\left\|m_{n}\right\|\right\} \in l^{1}$. For each positive integer $n$ and each increasing member $f$ of $C(X)$ we have $\int f d m_{n}=\int f e_{n} d m \geqq 0$. By Nachbin's Theorem, there exists a $\mu_{n} \in M_{\tau}(B o(G-\Delta)), \mu_{n} \geqq 0,\left\|\mu_{n}\right\|=$ $\left\|m_{n}\right\| / 2$, such that $\int_{X} f d m_{n}=\int_{G-\Delta} f^{*} d \mu_{n}$ for each $f \in C(X)$. For each $n$, let $\varphi_{n}: E \rightarrow R,\left(s_{k}\right) \mapsto s_{n}$. For each $s \in E$, the series $\sum_{n=1}^{\infty} \varphi_{n}(s) \mu_{n}$ converges in $M_{\tau}(B o(G-\Delta))$ since $\Sigma\left|\varphi_{n}(s)\right|\left\|\mu_{n}\right\| \leqq\|s\|_{p} \Sigma\left\|\mu_{n}\right\|<\infty$ and the space $M_{\tau}(B o(G-\Delta))$ is a Banach space under the total variation norm. Define $\mu: B o(G-\Delta) \rightarrow E^{\prime}$ by

$$
\mu(A) s=\sum_{n=1}^{\infty} \varphi_{n}(s) \mu_{n}(A)
$$

For each $s \in E$, we have $\mu s=\sum \varphi_{n}(s) \mu_{n} \in M_{\tau}(B o(G-\Delta))$. Let $A_{1}, \cdots, A_{n}$ be a finite partition of $G-\Delta$ into Borel sets and $s_{1}, \cdots, s_{n} \in E$ with $\left\|s_{i}\right\|_{p} \leqq 1$, then

$$
\begin{gathered}
\sum_{j=1}^{n}\left|\mu\left(A_{\jmath}\right) s_{\jmath}\right|=\sum_{j=1}^{n}\left(\left|\sum_{k=1}^{\infty} \varphi_{k}\left(s_{\jmath}\right) \mu_{k}\left(A_{j}\right)\right|\right) \leqq \sum_{j=1}^{n} \sum_{k=1}^{\infty} \mu_{k}\left(A_{\jmath}\right) \\
\sum_{k=1}^{\infty}\left(\sum_{j=1}^{n} \mu_{k}\left(A_{j}\right)\right)=\sum_{k=1}^{\infty}\left\|\mu_{k}\right\|=\sum_{n=1}^{\infty}\left\|m_{n}\right\| / 2<\infty .
\end{gathered}
$$


This proves that $\mu \in M_{\tau}\left(B o(G-\Delta), E^{\prime}\right)=M_{t}\left(B o(G-\Delta), E^{\prime}\right)$ where the last equality holds since $G-\Delta$ is locally compact. Finally, let $f \in$ $C(X, E)$ and put $f_{n}=\varphi_{n} \circ f$. Then

$$
f=\sum_{n=1}^{\infty} f_{n} e_{n} \quad \text { and } \quad f^{*}=\sum_{n=1}^{\infty} f_{n}^{*} e_{n} .
$$

Therefore,

$$
\begin{aligned}
\int_{X} f d m & =\lim _{N \rightarrow \infty} \sum_{n=1}^{N} \int f_{n} d\left(m e_{n}\right)=\lim _{N \rightarrow \infty} \sum_{n=1}^{N} \int_{G-\Delta} f_{n}^{*} d \mu_{n} \\
& =\lim _{N \rightarrow \infty} \sum_{n=1}^{N} \int_{G-\Delta} f_{n}^{*} e_{n} d \mu=\int_{G-\Delta} f^{*} d \mu .
\end{aligned}
$$

Hommel has shown in [4] that if $m$ is an increasing Radon measure on a locally compact space $X$ and if $\mu \geqq 0$ is a Radon measure on the graph $G$ of the order of $X$ such that $\int_{X} f d m=\int_{G} f^{*} d \mu$ for each $f \in C^{b}(X)$, then for each compact subset $K$ of $X$ we have

$$
\mu((K \times K) \cap G)+\|\mu\| \geqq|m|(K) .
$$

The following Theorem gives a similar result for operator valued measures.

THEOREM 2.7. Let E be a Banach lattice with a unit element $e$ and let $p$ denote the norm of $E$. Then:

(1) If $m \in M\left(B(X), E^{\prime}\right)$ and if $\mu \geqq 0$ is a member of $M\left(B(G), E^{\prime}\right)$ such that $\int_{X} f d m=\int_{G} f^{*} d \mu$ for each $f \in C_{r c}(X, E)$, then

$$
\|\mu\|_{p}+\mu_{p}((V \times V) \cap G) \geqq m_{p}(V)
$$

for each cozero set $V$ of $X$.

(2) If $m \in M_{t}\left(B o(X), E^{\prime}\right)$ and if $0 \leqq \mu \in M_{\tau}\left(B o(G), E^{\prime}\right)$ is such that $\int_{X} f d m=\int_{G} f^{*} d \mu$ for each $f \in C_{r c}(X, E)$, then $(*)$ holds also if $V$ is open or compact.

Proof. Let $m, \mu$ be as in (1) and let $V$ be a cozero subset of $X$. By [8], Theorem 2.2, we have

(a) $m_{p}(V)=\sup \left\{\int f d m: f \in C_{r c}(X, E),\|f\| \leqq 1, f=0\right.$ on $\left.X-V\right\}$. 
Let now $f \in C_{r c}(X, E)$ with $\|f\| \leqq 1$ and $f=0$ on $X-V$. Put $g=$ $\sup \left\{0, f^{*}-e\right\}$. Then $0 \leqq g \leqq e, \quad f^{*}-g \leqq e \quad$ and $g=0 \quad$ on $G-(V \times V) \cap G$. Hence,

$$
\int_{G} g d \mu \leqq \int_{(V \times V) \cap G} e d \mu=\mu_{p}((V \times V) \cap G)=a .
$$

Thus,

$$
\begin{aligned}
\int_{X} f d m-a & =\int_{G} f^{*} d \mu-a \leqq \int_{G}\left(f^{*}-g\right) d \mu \\
& \leqq \int_{G} e d \mu=\|\mu\|_{p} .
\end{aligned}
$$

Now the $(*)$ follows from (a).

Next, assume that $m, \mu$ are as in (2). Then (a) also holds for each $V$ open in $X$. Now, the same argument which was used to prove (1), shows that $(*)$ holds for each $V$ open. Finally in the same case, let $K$ be a compact subset of $X$. Given $\epsilon>0$, there exists an open subset $W$ of $G$ containing $(K \times K) \cap G$ and such that $\mu_{p}(W)<b+\epsilon$, where $b=$ $\mu_{p}((K \times K) \cap G)$. Let $V_{1}$ be an open subset of $X$ such that $V_{1} \cap G=$ $W$. The set $V_{2}=V_{1} \cup(X-G)$ is open in $X$ and $V_{2} \cap G=W$. Since $K \times K \subset V_{2}$ and $K$ is compact, there exists $V \supset K$ open such that $V \times V \subset V_{2}$. Now

$$
\begin{aligned}
\|\mu\|_{p}+\mu_{p}((K \times K) \cap G) & \geqq\|\mu\|_{p}+\mu_{p}((V \times V) \cap G)-\epsilon \\
& \geqq m_{p}(V)-\epsilon \geqq m_{p}(K)-\epsilon
\end{aligned}
$$

which completes the proof.

\section{Linear operators from $C(X)$ into $E$ positive on increasing functions.}

THEOREM 3.1. Let $X$ be a compact ordered space and let $C(X)$ be equipped with the uniform norm topology. Let $\varphi: C(X) \rightarrow E$ be a continuous linear map which is positive on the increasing members of $C(X)$. Then, the following are equivalent:

(1) There exists a positive linear map $\Phi: C(G) \rightarrow E$ such that $\Phi\left(f^{*}\right)=\varphi(f)$ for each $f \in C(X)$.

(2) There exists $u \geqq 0$ in $E$ such that $\varphi(f) \in[-u, u]$ for each $f \in C(X)$ with $\|f\| \leqq 1$.

If the order interval $[-u, u]$ of (2) is weakly compact, then there exists $\mu: B o(G) \rightarrow E$ positive such that: 
(a) For each $x^{\prime} \in E^{\prime}, x^{\prime} \circ \mu \in M_{\tau}(B o(G))$.

(b) For every bounded Borel function $f: G \rightarrow R$ and any $A \in$ $B o(G)$ there exists an element of $E$, denoted by $\int_{A} f d \mu$, such that

$$
x^{\prime}\left(\int_{A} f d \mu\right)=\int_{A} f d\left(x^{\prime} \circ \mu\right)
$$

for each $x^{\prime} \in E^{\prime}$.

(c) $\int_{G} f^{*} d \mu=\varphi(f)$ for each $f \in C(X)$.

Proof. $(1 \Rightarrow 2)$. Let $\Phi$ be as in (1) and let $f \in C(X),\|f\| \leqq$ 1. Then $-1 \leqq f^{*} / 2 \leqq 1$ and hence $-\Phi(1) \leqq \varphi(f) / 2 \leqq \Phi(1)$.

$(2 \Rightarrow 1)$. Let $u \in E$ be as in (2). Let $f \in C(X)$ with $f^{*} \leqq 1$. If $x \leqq y$, then $(-f)(x) \leqq(-f)(y)+1$. By Nachbin [11], Theorem 6, there exists an increasing member $g$ of $C(X)$ such that $\|g+f\| \leqq 1 / 2$. Hence

$$
\varphi(f)=\varphi(f+g)-\varphi(g) \leqq \varphi(g+f) \leqq u / 2 .
$$

Let $G_{1}$ be the subspace of $C(G)$ spanned by the set

$$
\left\{f^{*}: f \in C(X)\right\} \cup\{1\} \text {. }
$$

On $G_{1}$, we define

$$
\Phi_{1}: G_{1} \rightarrow E, \quad f^{*}+\lambda \mapsto \varphi(f)+\lambda u / 2 .
$$

Then $\Phi_{1}$ is well defined, it is linear and positive. In fact, if $f^{*}+\lambda \geqq 0$ (where $f \in C(X)$ and $\lambda \in R$ ), then $\lambda \geqq 0$ because $f^{*}=0$ on $\Delta$. If $\lambda>0$, then $-f^{*} / \lambda \leqq 1$ and thus $\varphi(-f / \lambda) \leqq u / 2$ which gives $\varphi(f)+\lambda u / 2 \geqq$ 0 . Also, if $\lambda=0$, then $f^{*} \geqq 0$. Thus, in this case, $f$ is increasing and so $\varphi(f) \geqq 0$. It follows that $\Phi_{1}\left(f^{*}+\lambda\right) \geqq 0$ if $f^{*}+\lambda \geqq 0$. Now, there exists (see Peressini [14], page 83, Proposition 2.9) a positive extension $\Phi$ of $\Phi_{1}$ to all of $C(G)$.

Finally, assume that the order integral $[-u, u]$ of $(2)$ is weakly compact. As in the proof of (2), there exists a positive linear map $\Phi: C(G) \rightarrow E$ such that $\Phi(1)=u / 2$ and $\Phi\left(f^{*}\right)=\varphi(f)$ for each $f \in C(X)$. If $g \in C(G)$ is such that $\|g\| \leqq 1$, then $-1 \leqq g \leqq 1$ and hence $\Phi(g) \in[-u / 2, u / 2]$. By hypothesis, the interval $[-u / 2, u / 2]$ is weakly compact. It follows that $\Phi$ is a weakly compact operator. By Lewis ([10], page 163, Theorem 3.1) there exists $\mu: B o(G) \rightarrow E$ having properties (a), (b) and such that

$$
\int_{G} f d \mu=\Phi(f)
$$


for each $f \in C(G)$. In particular (c) holds. It remains to show that $\mu(A) \geqq 0$ for each Borel subset $A$ of $G$. To this end, assume first that $A$ is a zero subset of $G$. There exists a sequence $\left(f_{n}\right) \subset C(G), 0 \leqq f_{n} \leqq 1$, $f_{n}=1$ on $A$ and $\left(f_{n}\right)$ decreases pointwise to the characteristic function of $A$. From the

$$
\Phi\left(f_{n}\right)=\int_{A} f_{n} d \mu+\int_{G-A} f_{n} d \mu=\mu(A)+\int_{G-A} f_{n} d \mu
$$

and from the fact that $\int_{G-A} f_{n} d \mu \rightarrow 0$ weakly in $E$, it follows that $\Phi\left(f_{n}\right) \rightarrow \mu(A)$ weakly. Since the positive cone of $E$ is convex and closed, it is weakly closed. Thus $\mu(A) \geqq 0$.

Assume next that $A$ is an element of $B(G)$. Given $x^{\prime} \in E^{\prime}$ and $\epsilon>0$, we can find, by the regularity of $x^{\prime} \circ \mu$, a zero set $Z \subset A$ such that $\left|x^{\prime} \circ \mu\right|(A-Z)<\epsilon$. Thus $\left|x^{\prime}(\mu(A)-\mu(Z))\right|<\epsilon$. This, by the first part of the proof, shows that $\mu(A)$ belongs to the weak closure of the positive cone of $E$ and hence it is positive.

Suppose now that $F$ is a closed subset of $G$ and let $x^{\prime} \in E^{\prime}$ and $\epsilon>0$. From the regularity of $x^{\prime} \circ \mu$ and from the fact that the cozero sets form a basis for the open subsets of $G$, we can find a cozero set $V \supset F$ such that $\left|x^{\prime}(\mu(V)-\mu(F))\right|<\epsilon$. This again proves that $\mu(F) \geqq 0$.

Finally, using again the regularity of $x^{\prime} \circ \mu$, for $x^{\prime} \in E^{\prime}$, we show that $\mu(A) \geqq 0$ for each $A \in B o(G)$ and this completes the proof.

Corollary 3.2. Let $X$ be a compact ordered space, E a Banach lattice with a unit element $e$ and $\varphi: C(X) \rightarrow E$ a continuous linear map such that $\varphi(f) \geqq 0$ for each increasing $f \in C(X)$. Then, there exists $a$ positive linear map $\Phi: C(G) \rightarrow E$ such that $\varphi(f)=\Phi\left(f^{*}\right)$ for each $f$ in $C(X)$. If $E$ is in addition reflexive, then there exists a positive $\mu: B o(G) \rightarrow E$ such that :

(a) For each $x^{\prime} \in E^{\prime}, x^{\prime} \circ \mu \in M_{\tau}(B o(G))$.

(b) For every bounded Borel function $f: G \rightarrow R$ and any Borel subset $A$ of $G$, there exists an element $\int_{A} f d \mu$ of $E$ such that $x^{\prime}\left(\int_{A} f d \mu\right)=$ $\int_{A} f d\left(x^{\prime} \circ \mu\right)$ for each $x^{\prime} \in E^{\prime}$.

(c) For each $f \in C(X)$, we have $\varphi(f)=\int_{G} f^{*} d \mu$.

Proof. If $\|f\| \leqq 1, f \in C(X)$, then $\varphi(f) \in[-u, u]$ where $u=$ $\|\varphi\| e$. Also, if $E$ is reflexive, then [ $-e, e]$ (being the closed unit ball of $E$ ) is weakly compact. Thus the result follows from the preceding Theorem. 


\section{REFERENCES}

1. R. G. Bartle, N. Dunford, and J. Schwatz, Weak compactness and vector measures, Canad. J. Math., 7 (1955), 289-305.

2. N. Bourbaki, Integration, Chap. I-IV, Act. Sci. et Ind. 1175, Paris 1965.

3. A. Grothendieck, Sur les applications linéaries faiblement compactes d'espaces du type $C(K)$, Canad. J. Math., 5 (1953), 129-173.

4. G. Hommel, Increasing Radon measures on locally compact ordered spaces, Rendiconti di Mat., 9 (1976), 85-117.

5. A. K. Katsaras, Spaces of vector measures, Trans. Amer. Math. Soc., 206 (1975), 313-328.

6. Continuous linear functionals on spaces of vector-valued functions, Bull. Soc. Math. Grèce, 15 (1974), 13-19.

7. _ Locally convex topologies on spaces of continuous vector functions, Math. Nach., 71 (1976), 211-226.

8. _ Vector-valued measures and strict topologies, Annali di Mat. IV, Vol. CVI (1975), 261-272.

9. A. K. Katsaras and D. B. Liu, Integral representations of weakly compact operators, Pacific J. Math., 56 (1975), 547-556.

10. D. R. Lewis, Integration with respect to vector measures, Pacific J. Math., 33 (1970), 157-165.

11. L. Nachbin, Linear continuous functionals positive on the increasing continuous functions, Sum. Bras. Math. II, Fasc., 10 (1951), 135-150.

12. - Sur les espaces uniformisables ordonnés, Comptes Rendus Acad. Sci. Paris, 226 (1948), 547.

13. Topology and Order, Van Nostrand, New York, 1965. Reprinted by Krieger, New Jersey, 1976.

14. A. L. Peressini, Ordered Topological Vector Spaces, Harper \& Row, New York, 1967.

15. J. Prolla, On the extension of increasing continuous functions, Bull. Soc. Roy. Sci. Liège, (to appear).

16. V. Varadarajan, Measures on topological spaces, Amer. Math. Soc. Transl., (2) 48 (1965), $161-228$.

Received November 29, 1976.

Univ. Estad. DE CAMPINAS

13100 Campinas, São Paulo

BRAZIL

Present address: Department of MATHEMATICS

UNIVERSITY OF IOANNINA

IOANNINA, GREECE 



\section{Pacific Journal of Mathematics \\ Vol. 70, No. $1 \quad$ September, 1977}

William H. Barker, Noether's theorem for plane domains with hyperelliptic

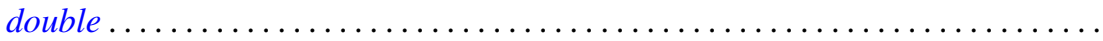

Michael James Beeson, Non-continuous dependence of surfaces of least area on the boundary curve ...................................... 11

Horst Behncke, Functions acting in weighted Orlicz algebras . . . . . . . . . . . . 19

Howard Edwin Bell, A commutativity study for periodic rings . . . . . . . . . . . 29

Peter Botta and Stephen J. Pierce, The preservers of any orthogonal group ....... 37

Douglas S. Bridges, The constructive Radon-Nikodým theorem ............. 51

James Dennis Brom, The theory of almost periodic functions in constructive

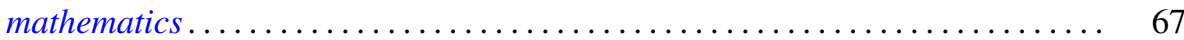

N. Burgoyne and C. Williamson, Semi-simple classes in Chevalley type groups ....

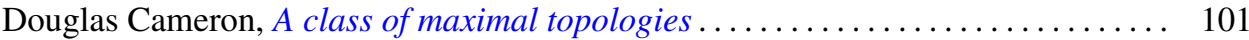

L. Carlitz, Enumeration of doubly up-down permutations . . . . . . . . . . . . . . 105

Paul Robert Chernoff, The quantum n-body problem and a theorem of

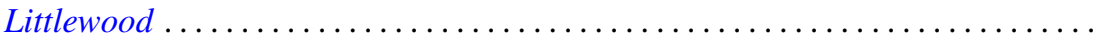

Jo-Ann Deborah Cohen, Locally bounded topologies on $F(X) \ldots \ldots \ldots \ldots \ldots \ldots$

Heinz Otto Cordes and Robert Colman McOwen, Remarks on singular elliptic

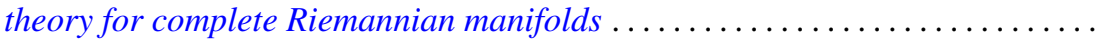

Micheal Neal Dyer, Correction to: "Rational homology and Whitehead

products"

Robert Fernholz, Factorization of Radonifying transformations

Lawrence Arthur Fialkow, A note on quasisimilarity. II ...... . .

Harvey Charles Greenwald, Lipschitz spaces of distributions on the surface of unit

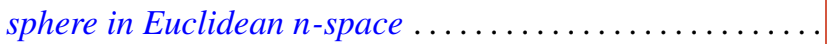

Albrecht Irle, On the measurability of conditional expectations

Tom (Roy Thomas Jr.) Jacob, Matrix transformations involving simple sequence

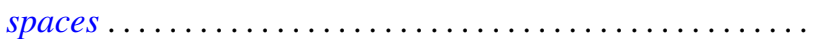

A. Katsaras, Continuous linear maps positive on increasing continuous

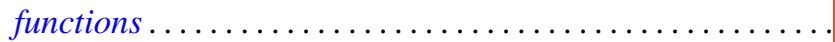

Kenneth Kunen and Judith Roitman, Attaining the spread at cardinals of cofinality

Lawrence Louis Larmore and Robert David Rigdon, Enumerating normal bundles

of immersions and embeddings of projective spaces ...... . .

Ch. G. Philos and V. A. Staïkos, Asymptotic properties of nonoscillatory solutions of differential equations with deviating argument .

Peter Michael Rosenthal and Ahmed Ramzy Sourour, On operator algebras containing cyclic Boolean algebras...

Polychronis Strantzalos, Strikt fast gleichgradig-stetige und eigentliche

Aktionen ...

Glenn Francis Webb, Exponential representation of solutions to an abstract

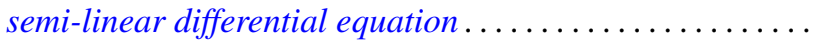

